\title{
Detecção Automática de Estilos de Aprendizagem utilizando Clusterização e Traços de Navegação
}

\author{
Rafael Miranda Abreu ${ }^{1}$, Cristiano Grijó Pitangui ${ }^{2}$, Luciana Pereira de Assis ${ }^{1}$, \\ Alessandro Vivas Andrade ${ }^{1}$, Cristiano Maciel da Silva ${ }^{2}$ \\ ${ }^{1}$ Universidade Federal dos Vales do Jequitinhonha e Mucuri \\ ${ }^{2}$ Universidade Federal de São João del-Rei \\ \{rafael.abreu, lpassis, alessandro.vivas\}@ufvjm.edu.br \\ \{pitangui.cristiano, cristiano\}@ufsj.edu.br
}

\begin{abstract}
Virtual Learning Environments are widely used to support the teaching-learning process, however, most of these systems provide the same content in the same way to all students. Recent studies point that the provision of personalized content to each student, through the detection of their Learning Style, greatly favors the teaching-learning process. This work proposes a fully automatic approach to identify students' Learning Styles through their interactions in Virtual Learning Environments. Preliminary results indicate that the proposed approach is promising in the automatic identification of students' learning preferences.
\end{abstract}

Resumo. Os Ambientes Virtuais de Aprendizagem são amplamente utilizados no apoio ao ensino-aprendizagem, contudo, a maioria destes sistemas fornece o mesmo conteúdo de iguais maneiras e formatos a todos os alunos. Há pesquisas que indicam que a oferta de conteúdo personalizado a cada aluno, por meio da detecção de seu Estilo de Aprendizagem, favorece, sobremaneira, o processo de assimilação do aluno. Este trabalho propõe uma abordagem totalmente automática para a detecção de Estilos de Aprendizagem de alunos por meio de suas interações em Ambientes Virtuais de Aprendizagem. Resultados preliminares apontam que a abordagem proposta mostra-se promissora na identificação automática das preferências de aprendizagem dos alunos.

\section{Introdução}

A Educação a Distância (EAD), além de permitir uma maior interação entre as pessoas, disponibiliza conteúdos a todos os usuários mesmo quando distantes geograficamente. Entretanto, como cita [Resende et al. 2014], existe uma desvantagem quanto à transmissão do ensino, pois o conteúdo é ofertado da mesma maneira a alunos com perfis semelhantes, ignorando, portanto, suas especificidades e preferências. Partindo deste princípio, é desejável que Instituições Educacionais personalizem seus processos de acordo com as características de seus estudantes [Kurilovas et al. 2014].

Desde que se começou utilizar tecnologias da informação na Educação, diversas ferramentas para auxílio ao ensino-aprendizagem foram implementadas, como exemplo, os Ambientes Virtuais de Aprendizagem (AVAs). [Ribeiro et al. 2013] descreve este tipo de ambiente como sendo sistemas computacionais virtuais, que estão disponíveis aos seus 
VII Congresso Brasileiro de Informática na Educação (CBIE 2018)

Anais do XXIX Simpósio Brasileiro de Informática na Educação (SBIE 2018)

usuários por meio da internet. Estes sistemas permitem integrar múltiplas mídias, ordenar informações, desenvolver interações entre pessoas e objetos de conhecimento.

AVAs geralmente utilizam-se de Objetos de Aprendizagem (OA) para auxílio ao ensino. OA podem ser definidos como entidades a serem utilizadas dentro do processo de aprendizagem [Zaina et al. 2012]. São exemplos de OA: vídeos, figuras, slides, exercícios, dentre outros. Uma vez que, de maneira geral, os AVAs ofertam o mesmo conteúdo de igual maneira a todos estudantes, é crescente o avanço de estudos sobre recomendação personalizada de Objetos de Aprendizagem [Dorça and Resende 2015].

De acordo com vários estudiosos, dentre eles [Rezende et al. 2015], para que um OA seja recomendado ao estudante de forma personalizada, suas características e preferências são fatores que devem ser levados em consideração. Ainda, segundo [Graf et al. 2008], para associar o conteúdo mais adequado a um aluno, primeiramente deve-se conhecer o seu Estilo de Aprendizagem (EA). [Borges and Stiubiener 2014] refere-se ao EA como sendo as preferências dos indivíduos em capturar, organizar e transformar a informação em conhecimento, de maneira a facilitar a compreensão.

Segundo [Khribi et al. 2009], existem duas maneiras de se descobrir o EA de um aprendiz, de forma colaborativa ou automática. A primeira requer o fornecimento explícito de informações por parte do aluno, geralmente obtidas pelo preenchimento de questionários. Na segunda, as informações são coletadas automaticamente de acordo com o comportamento online do usuário em um AVA. Neste contexto, [Graf et al. 2008] ressalta que utilização de questionários não é confiável, pois o aluno pode respondê-lo equivocadamente por falta de compreensão, ou até mesmo por motivos emocionais. Por sua vez, a abordagem automática é mais precisa e menos propensa a erros, pois esta analisa os dados comportamentais reais do aluno [Khribi et al. 2013].

Neste sentido, e com base no modelo de Estilo de Aprendizagem de [Felder and Silverman 1988], este trabalho apresenta uma abordagem capaz de detectar automaticamente o EA de estudantes por meio da utilização de técnicas de Mineração de Dados (MD) em bases de dados AVA Moodle. Devido à baixa representação de alguns EA em bases de dados reais, apresenta-se um simulador de alunos que emula o comportamento dos mesmos em AVA de acordo com seus EA. O simulador foi utilizado para gerar uma base de dados artificial a qual contém alunos de todos possíveis tipos de EA de acordo com [Felder and Silverman 1988]. Resultados preliminares apontam que a abordagem proposta mostra-se promissora na identificação automática de EA.

O restante deste trabalho organiza-se como segue. A seção 2 apresenta o referencial teórico e os principais trabalhos relacionados a esta pesquisa. A seção 3 apresenta a abordagem proposta para a detecção automática de EA. Resultados preliminares são analisados na seção 4. Por fim, a seção 5 conclui o trabalho e aponta trabalhos futuros.

\section{Referencial Teórico}

\subsection{Ambientes Virtuais de Aprendizagem}

Ambientes Virtuais de Aprendizagem (AVA) são sistemas computacionais que permitem integrar diversas mídias, ordenar informações, e desenvolver interações entre seus usuários [Ribeiro et al. 2013]. Desde que começaram a ser utilizados na EAD, diversos destes sistemas surgiram, começando pelos ambientes clássicos, considerados limitados 
VII Congresso Brasileiro de Informática na Educação (CBIE 2018)

Anais do XXIX Simpósio Brasileiro de Informática na Educação (SBIE 2018)

por apresentar o mesmo conteúdo a todos estudantes e não possuírem uma assistência personalizada e inteligente [Resende et al. 2014].

Com o avanço tecnológico e a necessidade de considerar as particularidades de cada indivíduo no processo de ensino-aprendizagem, surgiram os sistemas de gerenciamento de aprendizagem, também conhecidos como Learning Management Systems (LMS). Para [Borges and Stiubiener 2014], estes softwares são utilizados para uma melhor comunicação entre aluno e professor e, especialmente, para fornecimento de instruções, materiais, avaliações e outros recursos que fornecem atividades colaborativas.

Dentre os LMSs existentes, o mais utilizado é o Moodle "Modular Object Oriented Dynamic Learning Environment", desenvolvido pelo australiano Martin Dougiamas na década de 90 . Um dos motivos dele ser ser bem aceito e difundido está no fato de que além de ser um sistema de código aberto, ainda é de fácil manuseio. Contudo, como apontato por [Ribeiro et al. 2013], o mesmo apresenta algumas dificuldades, pois, por muitas vezes, oferece o mesmo conteúdo da mesma maneira a todos os alunos.

Um dos objetivos de um AVA é fornecer materiais didáticos aos alunos. No entanto, a criação destes materiais demanda disponibilidade de tempo, além de conhecimentos tecnológicos. Neste sentido, e pensando-se em reutilização de material, surge o conceito de Objetos de Aprendizagem (OA) [Dorça and Resende 2015], definidos por [Zaina et al. 2012] como entidades a serem utilizadas dentro do processo de ensinoaprendizagem, e cita como exemplos: vídeos, figuras, simuladores, etc.

\subsection{Estilos de Aprendizagem e o modelo de Felder e Silverman}

É desejável que os AVAs sejam personalizados de acordo com as preferências do aprendiz [Kurilovas et al. 2014]. Para [Kurilovas et al. 2014], as Instituições Educacionais devem personalizar seu processo de aprendizagem de acordo com as principais características dos seus alunos, pois estes possuem diferentes necessidades e características, como conhecimento prévio, nível intelectual, traços cognitivos e Estilos de Aprendizagem (EA).

[Borges and Stiubiener 2014] refere-se ao EA como sendo as preferências dos indivíduos em capturar, organizar e transformar informações em conhecimento, de maneira a facilitar a compreensão do mesmo. Neste sentido, a adoção de EA específicos a cada perfil permite ao professor trabalhar com as diferentes habilidades dos alunos e, portanto, auxilia sobremaneira o processo de ensino-aprendizagem [Zaina et al. 2012].

[Dorça et al. 2016] cita alguns exemplos de modelos de estilo de aprendizagem: Kolb, Honey e Mumford, Entwistle, Pask, Felder e Silverman. Cada um destes e muitos outros existentes descrevem diferentes aspectos das preferências de aprendizado de cada aluno. De forma geral, modelo de Felder e Silverman (FSLSM - Felder and Silverman Learning Style Model) é o mais utilizado para a área educacional [Zaina et al. 2012].

Na visão de [Felder and Silverman 1988], a aprendizagem é um processo de duas fases que envolve a recepção e o processamento da informação. Neste modelo, as maneiras de como as informações são recebidas e processadas estão divididas em quatro dimensões, como ilustra a Figura 1.

A partir desse modelo, [Felder and Soloman 1991] criaram o Index of Learning Styles (ILS), um instrumento (questionário) para identificar o EA que contempla as dimensões contidas no modelo de Felder e Silverman. 


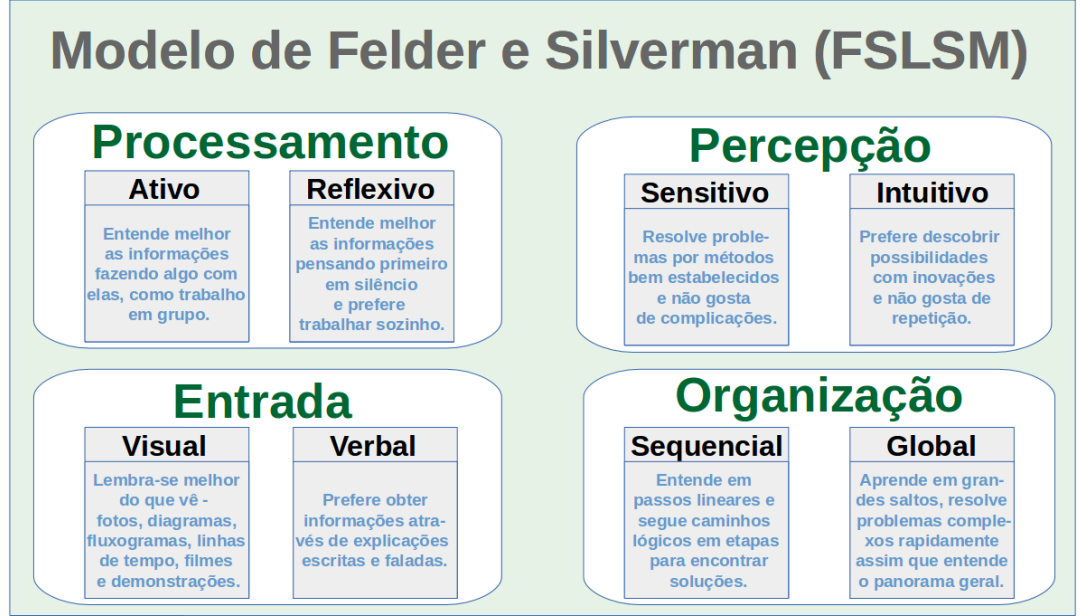

Figura 1. Dimensões do FSLSM - Fonte: [Kolekar et al. 2017] adaptado.

\subsection{Detecção de Estilos de Aprendizagem}

Estudos revelam que se um conteúdo for apresentado ao aluno de forma personalizada de acordo com suas preferências e características individuais, a tendência é que este conteúdo seja assimilado mais facilmente. Neste sentido, uma das formas de se realizar tal personalização é por meio da detecção do EA do aluno. Com esta finalidade, técnicas de modelagem de alunos estão sendo discutidas e Sistemas Adaptativos, bem como abordagens para detecção de EAs, estão sendo apresentadas e discutidas [Graf and Kinshuk 2009].

Como citado anteriormente, existem duas maneiras de descobrir o EA de um aprendiz, a primeira é de forma colaborativa, que requer o fornecimento explícito de informações por parte do usuário, e, na segunda, as informações do usuário são coletadas de forma automática de acordo com seu comportamento online [Khribi et al. 2009].

Atualmente, a maioria dos Sistemas Adaptativos que se concentra em EA usa a abordagem colaborativa, quase sempre solicitando aos alunos que preencham um questionário para detectar seus EA, ou para validar suas abordagens, como em [Bernard et al. 2015]. No entanto, para [Graf et al. 2008], a utilização desses questionários não é confiável, uma vez que o aluno pode respondê-lo de forma equivocada por falta de compreensão, ou até mesmo por motivos emocionais.

Alguns trabalhos, a exemplo de [Kirschner 2017], criticam a utilização de EA, no entanto, existem diversos outros trabalhos que utilizam diferentes técnicas para descobrir o EA de estudantes, como os citados a seguir.

[Bernard et al. 2017] procura melhorar a precisão de abordagens automáticas para detecção de EA. Para isso, apresenta 4 técnicas, cada uma baseada em um "tipo" de algoritmo: Rede Neural, Algoritmo Genético, Colônias de Formigas, e Otimização por Enxame de Partículas. As abordagens foram avaliadas com dados do Moodle e obtiveram resultados promissores. No entanto, apesar de serem abordagens automáticas, o questionário ILS foi aplicado para coletar dados de EA e realizar a validação dos resultados.

[Kolekar et al. 2017] propõe utilizar o algoritmo de clusterização (Fuzzy C Means) (FCM) para agrupar os dados comportamentais capturados nas categorias FSLSM, e depois gera-se um classificador a partir do algoritmo de classificação Gravitational Se- 
VII Congresso Brasileiro de Informática na Educação (CBIE 2018)

Anais do XXIX Simpósio Brasileiro de Informática na Educação (SBIE 2018)

arch based Back Propagation Neural Network (GSBPNN), para predizer o EA do aluno. Para capturar os dados de log dos alunos, um portal de e-learning foi desenvolvido. Os resultados obtidos pelo algoritmo GSBPNN são comparados aos de uma Rede Neural padrão (que utiliza o Back Propagation para seu treinamento). Resultados apontam que o desempenho do algoritmo GSBPNN é superior ao da Rede Neural padrão.

[Pitigala Liyanage et al. 2016] utiliza dados do Moodle para prever o EA dos alunos considerando o FSLSM. Os dados coletados foram trabalhados no framework WEKA (Waikato Environment for Knowledge Analysis) [Frank et al. 2016], que implementa os quatro algoritmos utilizados pelos autores, a saber: J48, Bayesian Network, Naive Bayes $e$ Random Forest. O questionário ILS foi aplicado para efeitos de avaliação e validação dos resultados. Adicionalmente, foi proposto um mapa de aprendizagem em grupo para auxiliar professores e alunos a alcançarem resultados de aprendizagem mais efetivos.

[Bernard et al. 2015] apresenta uma abordagem mais geral a fim de ser compatível com diferentes AVAs. A proposta é baseada no modelo FSLSM e utiliza quatro Redes Neurais, uma para cada dimensão do Estilo de Aprendizagem, para identificar os EA dos alunos. A abordagem foi avaliada utilizando dados de 75 alunos e o ILS foi utilizado para validação dos resultados. Apresenta-se que a precisão do método proposto supera as abordagens consideradas estado da arte.

[Paireekreng and Prexawanprasut 2015] propõe um modelo de classificação de EA onde técnicas de Classificação de Conjuntos foram implementadas. Aplicou-se o questionário ILS a 400 participantes. Destes, 300 registros foram utilizados como dados de treinamento, e 100 registros foram usados como dados de teste. As técnicas de classificação aplicadas foram Árvore de Decisão, Naive Bayes, Redes Neurais e Support Vector Machine (SVM). Utilizou-se um modelo de Classificação de Conjuntos para melhorar o desempenho por meio da implementação de um algoritmo de votação. Os resultados mostram que as técnicas de Classificação de Conjunto apresentam melhor desempenho em relação a outras técnicas de classificação utilizadas isoladamente.

[Khribi et al. 2013] apresenta uma abordagem de modelagem automática do aluno utilizando técnicas de MD na base de dados de um LMS. É aplicado um modelo hierárquico de multi-nível com base na abordagem de Filtragem Colaborativa, a fim de agrupar alunos com características, interesses, e preferências semelhantes. Cada classe obtida corresponde a alunos com a mesma dimensão do FSLM. Para implementar a abordagem, utilizaram-se os algoritmos $K$-way e Apriori. A abordagem está sendo aplicada no Moodle, no contexto de um sistema de recomendação que visa recomendar automaticamente links para os alunos, considerando suas preferências educacionais, incluindo seus EA.

Conforme apresentado, a maioria das pesquisas que trata da descoberta de Estilos de Aprendizagem utiliza a abordagem colaborativa, a qual necessita de informações explicitamente fornecidas por parte do estudante. Como já apontado, métodos que optam por esse tipo de abordagem estão mais sujeitos a falhas, o que torna as abordagens automáticas mais confiáveis. No entanto, a maioria das abordagens ditas automáticas, em algum momento, utiliza dados extraídos de questionários, seja para alimentar e treinar seus algoritmos, ou para validar os resultados obtidos. Possivelmente, devido a dificuldade em realizar essa validação, pesquisas de abordagens totalmente automáticas para detecção de EA ainda são bastante escassas, tornando a abordagem proposta diferenciada. 
VII Congresso Brasileiro de Informática na Educação (CBIE 2018)

Anais do XXIX Simpósio Brasileiro de Informática na Educação (SBIE 2018)

\section{Abordagem Proposta}

A fim de detectar o Estilo de Aprendizagem de estudantes sem que estes forneçam qualquer informação (mesmo para efeitos de validação), elaborou-se a abordagem automática apresentada na Figura 2.

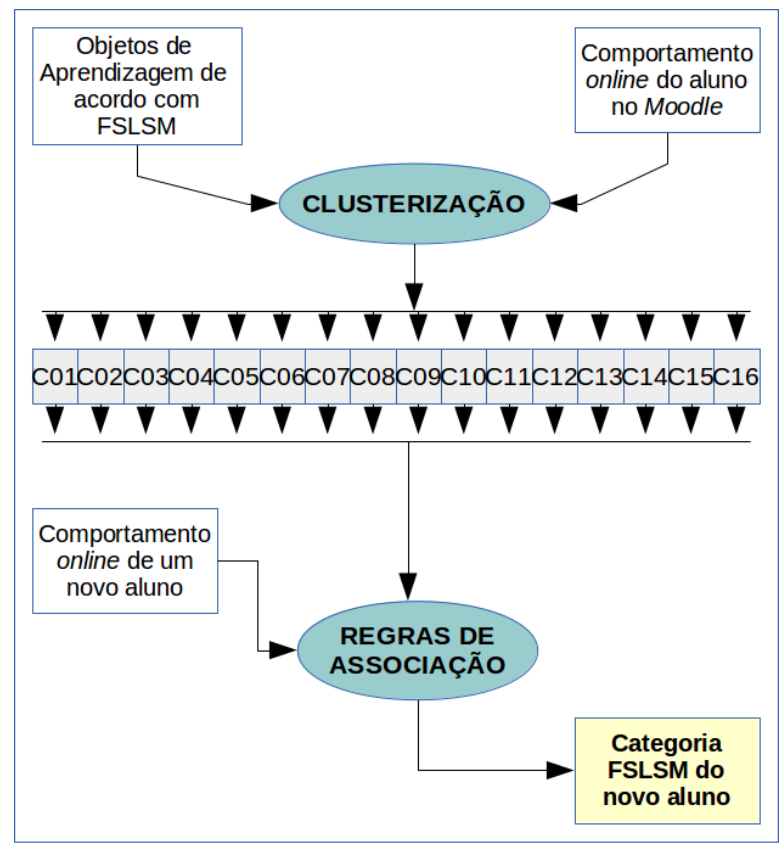

Figura 2. Abordagem proposta - Fonte: Próprio Autor.

Inicialmente, realiza-se a categorização das ferramentas e OA do Moodle de acordo com as dimensões do modelo FSLSM, como por exemplo: alunos ativos preferem postar em fóruns com mais frequência, aprendizes verbais visitam material de leitura com maior frequência, e assim por diante. Esta categorização é baseada nos trabalhos [Graf and Kinshuk 2007] e [Graf and Liu 2009].

Em seguida, realiza-se a extração de dados dos alunos que já estão registrados na base de dados do Moodle por meio de logs. De posse das informações obtidas com a categorização (nas dimensões do FSLSM) das ferramentas e OA presentes no Moodle, e dos dados pré-processados extraídos da base de dados do Moodle, realiza-se uma clusterização, onde os alunos serão agrupados de acordo com seu comportamento no Moodle, i.e., alunos que se comportam de maneira semelhante serão agrupados em um mesmo cluster. Conforme ilustrado na Figura 1, o FSLSM possui 8 categorias, sendo 2 em cada dimensão. Dessa forma, o aluno tem a possibilidade de se enquadrar em uma das 16 possíveis sequências de Estilos de Aprendizagem. Este é o motivo pelo qual optou-se pela clusterização em 16 grupos distintos.

O último passo da abordagem proposta gera um classificador utilizando o algoritmo Classification Based on Associations (CBA) que utiliza regras de associação como modelo para predizer o EA de um novo aluno. Neste sentido, as regras de associação geradas pelo CBA classificarão um novo aluno em um dos 16 clusteres. A escolha do CBA deve-se à facilidade de compreensão das regras geradas e também ao fato de que ele se mostra bastante competitivo em relação aos outros classificadores, inclusive quando aplicado a bases de dados Educacionais [Fernandes et al. 2017]. 
VII Congresso Brasileiro de Informática na Educação (CBIE 2018)

Anais do XXIX Simpósio Brasileiro de Informática na Educação (SBIE 2018)

É importante notar que, atualmente, bases de dados reais dificilmente contemplam os 16 possíveis tipos de EA descritos no FSLSM, uma vez que isto exigiria a utilização dos mais variados e diversos OA presentes no Moodle. Tal fato pode ser verificado uma vez que os trabalhos que investigam o tema abordado utilizam, de forma geral, dimensões isoladas do FSLSM, tal como [Liyanage et al. 2014] e [Leon and Popescu 2013]. Neste sentido, este trabalho propõe um simulador de alunos. Esta ferramenta possibilita a geração de alunos pertencentes a qualquer EA no FSLSM, o que possibilita realizar o treinamento dos algoritmos de classificação antecipando o ponto que em um futuro próximo, os mais diversos conteúdos estarão disponíveis nos mais variados OA, e, portanto, ter-se-ão bases de dados reais muito mais completas no sentido de contemplarem, se não todos, a maioria dos EA presentes no FSLSM.

\subsection{Simulador de Alunos}

A ferramenta implementada tem a função de gerar bases de dados artificiais que simulam bases reais de AVAs. Seu desenvolvimento baseou-se no estudo realizado por [Graf and Kinshuk 2007] e [Graf and Liu 2009], que definiram padrões relevantes de comportamento do estudante para cada dimensão do FSLSM. Assim, o simulador permite gerar alunos pertencentes a quaisquer categorias do FSLSM.

Cada estudante gerado é representado por valores que retratam interações com os diversos OA disponíveis no Moodle, tais como: número de postagens em fóruns, tempo utilizado na leitura de resumos, número de revisões nos testes de auto-avaliação, desempenho em questões específicas e gerais, número de OA ignorados, dentre outros. Foram considerados 32 atributos e os valores a eles atribuídos, que representam interações com o AVA, respeitam os limites especificados em [Graf and Kinshuk 2007] e [Graf and Liu 2009].

A título de exemplificação, considere dois dos atributos da ferramenta fórum, a saber: número de visitas e número de postagens. Alunos ativos preferem postar com mais frequência para perguntar, discutir e explicar questões sobre o material aprendido, enquanto alunos reflexivos participam de forma passiva lendo cuidadosamente e com frequência as postagens. Com relação aos limites, 50 ou mais visitas ao fórum, 10 ou mais postagens, podem ser consideradas como um comportamento acima da média [Graf and Kinshuk 2007] e [Graf and Liu 2009]. Assim, um aluno ativo teria, por exemplo, 55 visitas ao fórum, e 17 postagens nesta ferramenta.

É importante notar que alunos de cursos EAD não interagem apenas com atributos relacionados ao seu EA. Isso deve-se a diversos fatores, tais como, a obrigatoriedade no uso de certas ferramentas, pois estas farão parte da avaliação da disciplina, ou, mais comumente, oferecimento de um conteúdo utilizando apenas um OA. Por este motivo, foram adicionados ruídos à geração das interações dos alunos, assim, ao se gerar uma determinada interação de um aluno de um determinado EA, algumas de suas interações são geradas referentes a outro EA.

\section{Resultados Experimentais}

O simulador de alunos desenvolvido foi usado para gerar uma base de dados de 800 alunos contemplando todas possíveis sequencias de EA. Neste sentido, foram gerados 50 alunos em cada um dos 16 EA. 
VII Congresso Brasileiro de Informática na Educação (CBIE 2018)

Anais do XXIX Simpósio Brasileiro de Informática na Educação (SBIE 2018)

A inserção de ruídos na geração das interações dos alunos possibilita a criação de diversos tipos de estudantes, desde aqueles mais fáceis de se detectar o EA, até aqueles onde a descoberta de seu EA é mais difícil. Assim, o simulador emula casos que podem ocorrer durante interações reais dos alunos do AVA. Nos testes efetuados, o simulador trabalhou com 12,5\% de ruído, assim, 4 dos 32 atributos são preenchidos com valores de interações referentes a outro EA que não a do aluno.

O algoritmo K-Means, implementado no Weka, foi parametrizado para gerar 16 clusters, i.e., um cluster para cada EA na base de dados gerada. A acurácia na geração dos clusters pôde ser calculada verificando-se as referências de cada cluster gerado em relação a cada uma das 16 classes (EA) por meio da contagem dos erros na atribuição de um aluno a um cluster.

O K-Means obteve uma acurácia de 76\%, classificando corretamente 608 dos 800 alunos. Ao realizar a clusterização, o algoritmo atribuiu 13 classes (EA) a 13 clusters $(81,25 \%)$, sendo que 3 classes o $K$-Means não identificou, como ilustra a Figura 3.

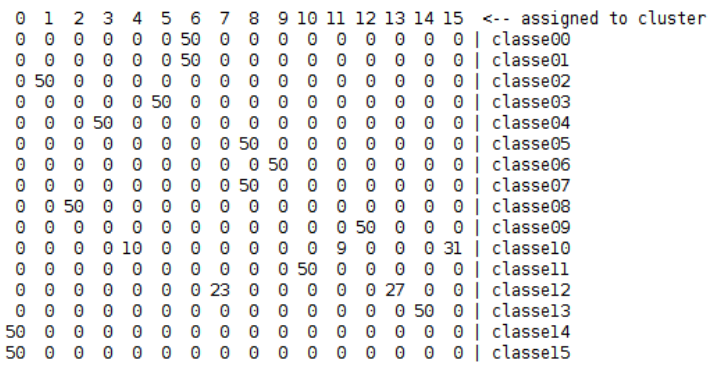

Figura 3. Resultados do WEKA - Fonte: Próprio Autor.

Das 13 classes (EA) identificadas, em 11 delas $(84,6 \%)$, os alunos foram corretamente classificados. Os 3 clusters que não foram associados a nenhuma classe (EA) representam erros de classificação do algoritmo. Ademais, o algoritmo confunde o EA de alguns alunos, como por exemplo, associa alunos da classe 01 ao cluster 6 , que representa a classe 00 .

Os resultados, ainda que preliminares, apontam que o $K$-Means apresenta-se como uma abordagem promissora na identificação de clusters de alunos que possuem um mesmo EA. Por outro lado, a não identificação de certos EA pelo $K$-Means, e a confusão de um EA com outro, são fatos sob investigação.

\section{Considerações Finais}

Este trabalho introduziu uma abordagem para detectar de forma totalmente automática o EA de alunos considerando o FSLSM. Para isso, propôs-se a aplicação das técnicas de clusterização e mineração de regras de associação nos logs do AVA Moodle.

Ademais, devido à baixa representação de alguns EA em bases de dados reais, introduziu-se um simulador de alunos que emula o comportamento dos mesmos de acordo com seus EA. Este simulador foi utilizado para gerar o comportamento de 800 alunos distribuídos nos 16 possíveis EA. O algoritmo de clusterização $K$-Means mostrou-se promissor na identificação de clusters de alunos que pertencem ao mesmo EA. 
VII Congresso Brasileiro de Informática na Educação (CBIE 2018)

Anais do XXIX Simpósio Brasileiro de Informática na Educação (SBIE 2018)

Como trabalhos futuros, pretende-se estudar aprofundadamente os erros de classificação cometidos pelo $K$-Means, bem como gerar regras de associação para classificação de alunos em bases de dados do AVA Moodle (passo final da abordagem proposta).

\section{Referências}

Bernard, J., Chang, T.-W., Popescu, E., and Graf, S. (2015). Using Artificial Neural Networks to Identify Learning Styles. Proceedings of the International Conference on Artificial Intelligence in Education, pages 541-544.

Bernard, J., Chang, T. W., Popescu, E., and Graf, S. (2017). Learning style Identifier: Improving the precision of learning style identification through computational intelligence algorithms. Expert Systems with Applications, 75:94-108.

Borges, G. and Stiubiener, I. (2014). Recommending learning objects based on utility and learning style. 2014 IEEE Frontiers in Education Conference (FIE) Proceedings, 2015-Febru(February):1-9.

Dorça, F. A., Araújo, R. D., de Carvalho, V. C., Resende, D. T., and Cattelan, R. G. (2016). An Automatic and Dynamic Approach for Personalized Recommendation of Learning Objects Considering Students Learning Styles: An Experimental Analysis. Informatics in Education, 15(1):45-62.

Dorça, F. A. and Resende, D. T. (2015). Recomendação de conteúdo personalizada com base em estilos de aprendizagem: uma abordagem prática. Revista Brasileira de Informática na Educação, 23(03):12.

Felder, R. and Silverman, L. (1988). Learning and teaching styles in engineering education. Engineering education, 78(June):674-681.

Felder, R. and Soloman, B. (1991). Learning Styles and Stratgies. Strategies, pages $10-12$.

Fernandes, W. L., Pitangui, C., Vivas, A., and Assis, L. (2017). Previsão de Desempenho de Estudantes usando o Algoritmo de Classificação Associativa. Anais dos Workshops do Congresso Brasileiro de Informática na Educação, page 734.

Frank, E., Hall, M. A., and Witten, I. H. (2016). The WEKA Workbench. Online Appendix for "Data Mining: Practical Machine Learning Tools and Techniques.

Graf, S. and Kinshuk (2007). Advanced Adaptivity in Learning Management Systems by Considering Learning Styles. In 2009 IEEE/WIC/ACM International Joint Conference on Web Intelligence and Intelligent Agent Technology, volume 3, pages 235-238. IEEE.

Graf, S. and Kinshuk (2009). Adaptivity in Learning Management Systems by Considering Learning Styles. In 2009 IEEE/WIC/ACM International Joint Conference on Web Intelligence and Intelligent Agent Technology, volume 3, pages 235-238. IEEE.

Graf, S., Kinshuk, and Liu, T. C. (2008). Identifying learning styles in learning management systems by using indications from students' behaviour. The 8th IEEE International Conference on Advanced Learning Technologies, pages 482-486.

Graf, S. and Liu, T.-C. (2009). Supporting Teachers in Identifying Students' Learning Styles in Learning Management Systems: An Automatic Student Modelling Approach. Educational Technology \& Society, 12(4):3-14. 
VII Congresso Brasileiro de Informática na Educação (CBIE 2018)

Anais do XXIX Simpósio Brasileiro de Informática na Educação (SBIE 2018)

Khribi, M. K., Jemni, M., Nasraoui, O., Graf, S., and Kinshuk (2013). Toward a Fully Automatic Learner Modeling Based on Web Usage Mining with Respect to Educational Preferences and Learning Styles. In 2013 IEEE 13th International Conference on Advanced Learning Technologies, pages 403-407. IEEE.

Khribi, M. K., Mohamed, J., and Olfa, N. (2009). Toward integrating the pedagogical dimension in automatic learner modeling within e-learning systems. Proceedings 2009 9th IEEE International Conference on Learning Technologies, pages 642-644.

Kirschner, P. A. (2017). Stop propagating the learning styles myth. Computers and Education, 106:166-171.

Kolekar, S. V., Pai, R. M., and Pai, M. (2017). Paper-Prediction of Learner's Profile based on learning styles in Adaptive E-learning System Prediction of Learner's Profile Based on Learning Styles in Adaptive E-learning System Paper-Prediction of Learner's Profile based on learning styles in Adaptive . 12(6).

Kurilovas, E., Zilinskiene, I., and Dagiene, V. (2014). Recommending suitable learning scenarios according to learners' preferences: An improved swarm based approach. Computers in Human Behavior, 30:550-557.

Leon, F. and Popescu, E. (2013). Exploring the Relationships between Students ' Learning Styles and Social Media Use in Educational Settings. Ieee, pages 657-662.

Liyanage, M. P. P., Gunawardena, K. S. L., and Hirakawa, M. (2014). Using Learning Styles to Enhance Learning Management Systems. for Chapter I, 07(02):1-10.

Paireekreng, W. and Prexawanprasut, T. (2015). An integrated model for learning style classification in university students using data mining techniques. In 2015 12th International Conference on Electrical Engineering/Electronics, Computer, Telecommunications and Information Technology (ECTI-CON), pages 1-5. IEEE.

Pitigala Liyanage, M. P., Gunawardena, K. L., and Hirakawa, M. (2016). Detecting Learning Styles in Learning Management Systems Using Data Mining. IPSJ Transactions on Computers and Education, 2(1):1-10.

Resende, D. T., Dorça, F. A., Cattelan, R. G., and Araújo, R. D. (2014). Em direção à recuperação automática de objetos de aprendizagem em repositórios através da associação dos estilos de aprendizagem de estudantes com metadados no padrão IEEE-LOM. Anais dos Workshops do Congresso Brasileiro de Informática na Educação, 3(1):445.

Rezende, P. A. A., Pereira, C. K., Campos, F., David, J. M. N., and Braga, R. (2015). PERSONNA: proposta de ontologia de contexto e perfil de alunos para recomendação de objetos de aprendizagem. Revista Brasileira de Informática na Educação, 23(01):70.

Ribeiro, F. A. A., Fonseca, L. C. C., and Freitas, M. D. S. (2013). Recomendando Objetos de Aprendizagem a partir das hashtags postadas no Moodle. XXIV Simpósio Brasileiro de Informática na Educação (SBIE 2013), 25(Cbie):82-91.

Zaina, L. A. M., Bressan, G., Cardieri, M. A. A. C., and Rodrigues Júnior, J. F. (2012). e-LORS: Uma Abordagem para Recomendação de Objetos de Aprendizagem. Revista Brasileira de Informática na Educação, 20(1):4. 